\title{
Molecular phylogeny of plant 14-3-3 proteins family
}

\author{
Mikhaylova Yu.V. ${ }^{1 *}$, Shishova M.F. ${ }^{2}$ \\ ${ }^{1}$ Komarov Botanical Institute Institute RAS, St. Petersburg, Russia \\ ${ }^{2}$ St. Petersburg State University, St. Petersburg, Russia \\ * email: YMikhaylova@binran.ru
}

14-3-3 proteins are conservative and wildly spread family of phosphate group-binding modulators required for protein-protein interaction. In plants 14-3-3 family members involved in regulation of such processes as cell division, growth and differentiation, as well as responses to different stress factors, implementation of hormone signalling and modification of metabolism. In the present work, we performed evolutionary analysis of 14-3-3 proteins in broad phylogenetic scale. We analysed 14-3-3 proteins in flowering plants including not only well studied model plants, but also important for understanding of angiosperm evolution species, like ancient basal angiosperm Amborella. We use for the analysis coding sequences of 14-3-3 genes, obtained from Phytozome 12 and GenBank NCBI databases. Sequences were aligned using MAFFT. Maximum likelihood (ML) phylogenetic analysis was performed using IQ-TREE algorithm. Sequence of yeast 14-3-3 protein BMH1 (UniProt A6ZRD4) was used as outgroup. 42 plant species from monocots, dicots and Amborella were sampled. Total number of 455 sequences broke into two major clades, which are 163 in epsilon-group and 292 in non-epsilon group of 14-3-3. The presence of both epsilon and non-epsilon isoforms in Amborella means that the split is ancient. Revealed diversification could take place in the early period of seed plants evolution. The observed distribution of isoforms in angiosperms species suggests that ancestral forms of epsilon, iota, psi and kappa 14-3-3 isoforms diverged in common ancestral of all flowering plants. Later in common ancestral of monocots and eudicots $\mathrm{mu}$ and omega groups segregated from epsilon and psi respectively. Subsequent whole genome duplication event formed variety of isoforms in different evolution lines of flowering plants. Therefore, in Amborella, that only species have no whole-genome duplication in its evolutionary history, there are only four isoforms of 14-3-3 protein. The opposite example is Brassicaceae, which experienced many rounds of whole genome duplications. As a result Arabidopsis has 13 isoforms of 14-3-3 proteins.

Acknowledgements: The work was supported by RFBI (No. 19-04-00655). 